Publications of the Astronomical Society of the Pacific, 113:403-404, 2001 March

(C) 2001. The Astronomical Society of the Pacific. All rights reserved. Printed in U.S.A.

\title{
Conference Highlights
}

\section{Ultracool Dwarf Stars: Surveys, Properties, and Spectral Classification ${ }^{1}$}

Although astronomers have been searching the skies for brown dwarfs for decades, it is only in the last few years, with more sensitive detectors and new near-infrared all-sky surveys, that these cool objects have popped out from the background. The first reliable detections came in 1995, with the identification of lithium and of methane. Lithium, a light alkali element, is depleted in objects more massive than 65 Jupiter masses, so identification of this line pegs the object as a brown dwarf. Methane on the other hand indicates that the photospheric temperature of the object is less than $1500 \mathrm{~K}$ and so by any models for low-mass objects is a brown dwarf. In the years since these exciting discoveries, large numbers of "ultracool dwarfs" (objects cooler than M dwarfs) have been found. Two new spectral types ( $\mathrm{L}$ and $\mathrm{T}$ ) have been proposed for the classification of these objects. Broadly speaking, L dwarfs are characterized by the progressive disappearance of the optical $\mathrm{TiO}$ and $\mathrm{VO}$ bands that dominate late $\mathrm{M}$ dwarfs and their replacement by metallic hydrides and neutral alkali metals. The $\mathrm{T}$ dwarfs on the other hand have infrared spectra that are dominated by methane. It is important to note that there is no astrophysical significance attached to the particular letters. In particular, it should be stressed that lithium detection is not essential to the $\mathrm{L}$ spectral class, nor does $\mathrm{L}$ define an object as being a brown dwarf.

We report on a 1 day meeting held under the auspices of the IAU Commissions 29 and 45 on 2000 August 12 at the 24th IAU General Assembly in Manchester, United Kingdom. The meeting, which was attended by over 70 delegates from a broad range of stellar disciplines, was convened in order to discuss the current status of research in the field of ultracool dwarfs, with special reference to the question of spectral classification.

The first session of the day was chaired by James Liebert (Steward Observatory) and was concerned with the question of theoretical model atmospheres of ultracool dwarfs. In his presentation Takashi Tsuji (University of Tokyo) showed his new hybrid models where the observed colors and spectra of both $\mathrm{L}$ and $\mathrm{T}$ dwarfs can be reasonably well reproduced by a unified set of models. In these the dust effects that dominate the spectra of $\mathrm{L}$ dwarfs and appear to be absent from $\mathrm{T}$ dwarfs can be explained if dust forms only deep in the photosphere, with the upper layers dominated by volatile molecules. Talks by both Adam Burrows (Steward Observatory)

\footnotetext{
${ }^{1}$ Conference was held in Manchester, England, United Kingdom, in 2000 August. The Proceedings will be edited by H. R. A. Jones and I. A. Steele and published in the Lecture Notes in Physics Series by Springer-Verlag.
}

and Yakiv Pavlenko (Main Astronomical Observatory of Ukrainian Academy of Sciences) stressed the importance of the alkali metal strengths. In particular, they noted that the strength of the line wings were a dominant effect in the spectra, meaning that the optical colors of ultracool dwarfs could be either red or blue(!). Burrows emphasized that for these strong resonance lines it is particularly important to include broadening due to collisional broadening. Pavlenko described investigations using various models which indicate that it is necessary to introduce a power-law opacity into the synthetic spectra to reproduce the overall shape of the observed spectra. This allows for a hotter synthetic temperature scale more in line with more empirical investigations. Pavlenko also presented synthetic spectra showing where potential signatures of deuterium in the lines of deuterated water HDO might be found. Tom Geballe (Gemini Observatory) presented spectra identifying weak methane features in L5 and L7 dwarf spectra and showing that any contamination from the Earth's atmosphere would produce much broader bands that observed. As with lithium in L dwarfs, these spectra caution against the idea that only $\mathrm{T}$ dwarfs contain methane. He also presented 1-2.5 $\mu \mathrm{m}$ spectra of objects from mid-L to $\mathrm{T}$ showing a spectral sequence with $\mathrm{CH}_{4}$ increasing as $\mathrm{CO}$ decreased $\left(\mathrm{H}_{2} \mathrm{O}\right.$ increasing in parallel with the methane bands).

The second session was chaired by Virginia Trimble (University of Maryland/ University of California, Irvine) and considered the observed properties of ultracool dwarfs. Keith Noll (Space Telescope Science Institute) described how a series of model 3-4 $\mu \mathrm{m}$ spectra of late $\mathrm{L}$ dwarfs had been constructed using a line list containing over $30,000 \mathrm{H}_{2} \mathrm{O}$ lines and 100,000 $\mathrm{CH}_{4}$ lines. This allowed the identification of the weak methane features in the L5 and L7 dwarf spectra presented by Geballe and the ruling out of other candidate absorbers such as HDO, $\mathrm{H}_{2} \mathrm{~S}$, and $\mathrm{HCl}$. Coryn Bailer-Jones (Max-Planck-Institut für Astronomie, Heidelberg) described a program of photometric monitoring of 17 late $\mathrm{M}$ and $\mathrm{L}$ dwarfs both in the field and in the Pleiades and $\sigma$ Ori clusters. Evidence for small $(<5 \%)$ variations was found in a number of the targets. The majority of the variations found were nonperiodic. John Gizis (California Institute of Technology) presented the results of a study of chromospheric activity in ultracool dwarfs. The frequency of $\mathrm{H} \alpha$ emission peaks at M7 and then decreases for cooler M and L dwarfs. The kinematics of the active ultracool objects were consistent with an old-disk stellar population, while the inactive objects were more typical of a younger population. This suggests that contrary to the usual age-activity relationship, low 
activity can be an indicator of youth (and hence substellar mass) in ultracool dwarfs. On the other hand, lithium detection is found to correlate well with kinematics $\left(v_{\text {tan }}\right)$ and supports the idea that it should be more prevalent at younger ages. Glenn Schneider (University of Arizona) described an imaging survey of 85 young, nearby stars using the coronagraph of the HST NICMOS camera to search for low-mass companions. A number of candidates had been found, which were being followed up astrometrically and spectroscopically with the HST STIS camera. Iain Steele (Liverpool John Moores University) described the results of a program of "optical" IZ photometry of L dwarfs, which showed that the color was a good indicator of spectral type all through the $\mathrm{M}$ and $\mathrm{L}$ dwarf regime and is therefore an efficient method of searching for ultracool objects without the expense of wide-field infrared surveys.

The final session of the day was chaired by Mike Bessell (Australian National University) and was concerned with the issue of spectral classification. Denise Stephens (New Mexico State University) showed that infrared spectral typing (as opposed to the harder to collect optical spectra) is consistent with those inferred from optical spectra. Observations were made at three wavelengths $\left(K, L_{s}\right.$, and $\left.L^{\prime}\right)$ of a sample of $\mathrm{L}$ and $\mathrm{T}$ dwarfs. The $L^{\prime}$ wavelength is of particular interest as it is not affected by methane. Hence the $K-L^{\prime}$ color varies between $\sim 1.0$ and $\sim 1.8$ between $\mathrm{L} 0$ and the $\mathrm{L} / \mathrm{T}$ transition objects. In contrast, the $K-L_{s}$ color varies between $\sim 0.1$ and $\sim 0.6$ for $\mathrm{L}$ dwarfs before the effect of methane in the $L_{s}$ spectra reduces this value to 0.2 for the L/T transition objects. Davy Kirkpatrick (California Institute of Technology) described his optical classification system for $\mathrm{L}$ dwarfs which is based on the strengths of lines and bands in the region 6300-10000 $\AA$. Objects of similar types were grouped together to give nine natural groups ranging from L0 to L8. The classification is therefore based solely on optical spectra morphology rather than any physical interpretation of the spectra. He commented that the lack of optical flux for $\mathrm{T}$ dwarfs means that this method of spectral classification is not realistic for lower temperature spectral types. He also indicated that the current 2MASS mass function in the ultracool dwarf regime has the form $d N / d M=M^{1 \pm 0.25}$. Rafael Rebolo (Instituto Astrofisica de Canarias), substituting for Eduardo Martin (University of Hawaii), described their work on the spectroscopy of substellar objects in the Pleiades and $\sigma$ Ori. Some of the $\sigma$ Ori objects could have masses below the deuterium-burning limit of $13 M_{J}$. This finding is similar to a number of other recent works showing that "free-floating" brown dwarfs are produced below the deuterium-burning limit. The observed spectra are compared with field objects to search for gravity-dependent features and were classified using an alternative classification scheme to that of Kirkpatrick, based on a physical interpretation of the spectra via comparison with model atmospheres. The final presentation was given by Adam Burgasser (California Institute of Technology), who described a spectral typing scheme for T dwarfs based on overall spectral morphology. The observed spectra were dominated by $\mathrm{H}_{2}, \mathrm{H}_{2} \mathrm{O}$, and $\mathrm{CH}_{4}$ in the near-infrared and $\mathrm{FeH}, \mathrm{H}_{2} \mathrm{O}$, and widely broadened $\mathrm{K} \mathrm{I}$ and $\mathrm{Na}$ I doublets in the optical.

The meeting was closed with a general discussion of the issues raised, including the questions of new mnemonics for the $\mathrm{O}$ to $\mathrm{T}$ spectral sequence, spectral typing beyond $\mathrm{T}$, the sharpness of the L/T dwarf transition, and the importance of the "missing" late L objects being found by the Sloan Digital Sky Survey. There was also some debate about the usefulness of the deuterium-burning limit as a possible dividing point between star and planet formation; however, the consensus seemed to be that there is currently little supporting observational evidence.

Iain A. Steele and Hugh R. A. Jones

Astrophysics Research Institute, Liverpool John Moores University, Twelve Quays House, Egerton Wharf,

Birkenhead CH41 1LD, England, UK 